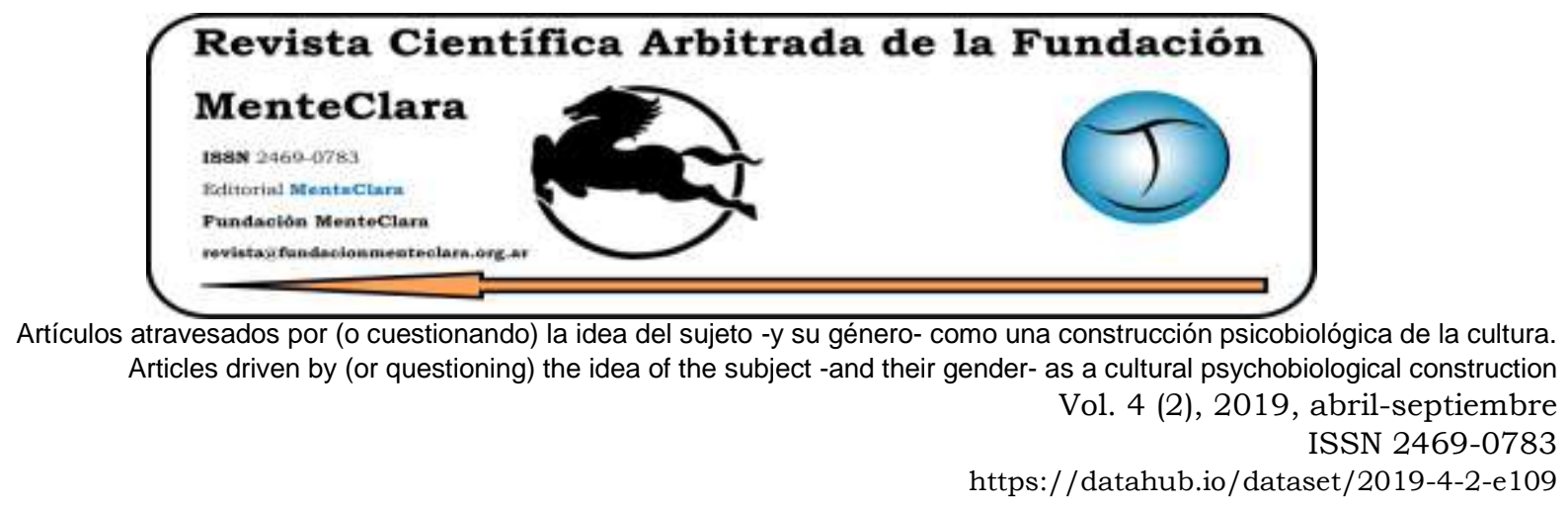

\title{
EDITORIAL VOL. 4 (2) 2019 - Cambio a publicación continua, políticas de revisión, artículos en EPUB y presentación del número.
}

\section{DOI: https://doi.org/10.32351/rca.v4.2.109}

Estimados lectores:

Continuando con nuestra meta de alcanzar el máximo nivel posible de una publicación académica incorporamos algunas modificaciones. La primera consiste en hacer disponible la totalidad de los artículos en formato epub. Desde el 2019, además de los formatos PDF, HTML y XML, los lectores dispondrán del formato EPUB para poder leer con independencia de nuestra plataforma y sin conexión, la totalidad de los artículos. Esto fue posible gracias a una alianza estratégico con ReadEra para ofrecer a nuestros lectores una herramienta gratuita y confiable, con la que leerlos en sus móviles. Esta alianza también nos permite replicar la totalidad de nuestros artículos en la plataforma de publicación internacional para ciencia, tecnología, medicina, educación y literatura de la Federación Rusa (https://readera.org).

Además les informamos que éste es el último número periódico. A partir de enero del 2020, nuestra publicación pasará a ser de semestral a publicación continua. De esta forma, el autor no tiene que esperar meses para que su trabajo sea publicado. A partir de este número los artículos se reciben, se evalúan y se publican inmediatamente, si son aceptados.

Otro problema que nuestro comité científico ha evaluado y tomado decisión es sobre la pregunta ¿Revisión a ciegas o revisión abierta?

Sabemos que la garantía de la ciencia es la participación de los evaluadores externos y que, estos revisores, tienen un papel central en la calidad de los trabajos. Este trabajo, en el proceso de revisión a ciegas -anónimo-, no le permite al profesional especializado que verifica entre otras cosas la metodología empleada en la investigación así como las referencias y el estado actual de la ciencia relacionada con 
el artículo, ningún tipo de crédito ya que la mayoría de las editoriales utilizan el trabajo voluntario de los revisores.

Varias editoriales, para compensar este anonimato, están utilizando el proceso de revisión abierta donde los revisores reciben crédito académico siendo sus revisiones publicadas en la revista, con DOI independiente, como único pago por su trabajo. Entendemos que es indigno que un revisor, que debe tener formación académica suficiente, trabaje solamente a cambio de un nuevo título publicado. Además, que el revisor sepa quién es el autor del artículo a revisar puede influir de manera inconsciente -negativa o positivamente- en su decisión. El siguiente ejemplo puede mostrar como el género de un investigador puede influir negativamente en su evaluador (Moss-Racusin et al, 2012).

Por estas razones concluimos que la tarea de revisor no debe ser gratuita y sumamos a nuestro presupuesto de financiación por artículo un valor de 80 euros para compensar esta tarea además de certificar su autoría de revisión en Publons luego de la publicación del artículo.

Para asumir estas fases de crecimiento de nuestra revista, que conlleva más trabajo y recursos económicos, queremos agradecer a Jorge Osvaldo Garcia, de la Universidad Nacional de la Plata, que suma su trabajo a nuestro Equipo Editorial.

Finalmente queremos presentarles los artículos de este número recorriendo el hilo conductor de nuestro enfoque editorial.

El primer artículo, (Duman, 2019) "Implementación del concepto de angst de Heidegger en el problema budista sobre el sufrimiento: Cómo el discurso budista genera dificultades epistemológicas y cómo abordarlo con la ontología occidentaln muestra como un investigador joven y brillante, guiado por Daniel Stephens de la Universidad Duke Kunshan de China, pudo descubrir a su corta edad como cambiando el término sufrimiento por angustia se puede esclavizar o liberar a los seguidores de una religión como el budismo. Nos muestra como un término polisémico como dukkha que en el budismo temprano aludia a la angustia existencial es prostituido y traducido como sufrimiento del que solamente se puede salir mediante una postura escatológica. Es decir, solamente por una liberación postmortem derivando en el budismo mahayana o theravada donde la "salvación" es individual y solo posible en otra "reencarnación" constituyéndose como una filosofia de opresión al igual que el sistema brahmánico 
colocando al budismo en la misma función que tiene el hinduismo según nos muestra Aparna Lanjewar Bose en el siguiente artículo:

"Hacia una poética comparativa de Buda, Kabir y Gurú Nanak desde una perspectiva democrática secular" (Bose, 2019) Este artículo muestra los valores democráticos desarrollados hace 2500 años por el Buda y más tarde en los siglos XIV y XV, por Kabir y Guru Nanak y explora el potencial emancipador y revolucionario de las creencias filosóficas y cosmovisiones igualitarias mostrando como la racionalidad, la igualdad, la fraternidad y la ideologia liberal predicada por ellos se encuentran en oposición binaria a la irracionalidad, la desigualdad y la ortodoxia prevalecientes hasta el presente en la India luego de la demonización de estas filosofias.

Seguidamente, Rudolph Bauer desmenuza esta filosofia que nos llega desde el budismo temprano y el budismo Vajrayana o esotérico -tántrico-, en los dos siguientes artículos: "La mente como conocedora de las formas y la conciencia como conocedora del Ser: Una visión fenomenológica" (Bauer, 2019 a) en este primer artículo, señala como el conocimiento de la filosofia tántrica incluía una forma de conocer la verdadera naturaleza de las cosas, la no dualidad del ser -es decir la inexistencia del alma- y fundamentalmente liberarse de este modo de la angustia ontológica, planteada por Ege Kaan Duman en el primer artículo de este número, mostrando cómo es posible salir del estado de dukkha. Seguidamente, este autor nos presenta en su segundo artículo: "Ontología de la dicha: Una fenomenología" (Bauer, 2019 b) En este artículo, Rudolf Bauer entrelaza e integra la ontología heideggeriana dentro de la experiencia de dicha como se documenta en las enseñanzas esotéricas del siglo XIX del maestro tántrico Dudjom Lingpa.

En el siguiente artículo: (Simkin et al, 2019) "Religiosidad y espiritualidad en el marco del modelo de los cinco factores de la personalidad" los autores describen el vínculo entre la religiosidad -las creencias- y los rasgos de la personalidad mostrando que se han reportado asociaciones positivas entre estos constructos como la apertura, la responsabilidad, la extroversión y la amabilidad y asociaciones negativas como el neuroiticismo. De esta forma se verifica como una creencia, una filosofia, puede elevar o deprimir a un sujeto.

Siguiendo en el terreno de verificar el modo en que una filosofia, una creencia, incide en el terreno de la salud presentamos el artículo (Dapena, 2019) "En busca de un posible tratamiento psicoanalitico de la ludopatian donde su autor, Jesús María 
Dapena Botero, busca un tratamiento que aborde al paciente desde la comprensión de sujeto no dual, es decir desde una visión contraria al dualismo cartesiano de mente y cuerpo, con su concepto de "Psiquesoma" equivalente a la visión de sujeto y su género como una construcción psicobiológica de la cultura de nuestro enfoque editorial.

En el artículo (Basu, 2019) "Soluciones al sufrimiento de los oprimidos: De la Revolución Cultural a la Evolución Cultural" el autor nos muestra cómo ni la filosofia de Adam Smith ni la filosofia hindú -sankhya- proporcionan ninguna guía para la transición eficaz de la psiquis humana hacia la evolución y muestra como ciertas filosofias igualitarias sí proponen un camino de evolución cultural por encima de las condiciones de nacimiento.

En el artículo "Modelos sociopoliticos en disputa. El caso del bloqueo a Huawei" (López Alurralde y Becerra, 2019) los autores presentan una breve comunicación, tomando como punto de partida el actual enfrentamiento comercial y tecnológico entre Estados Unidos y Huawei, para identificar y caracterizar dos modelos sociopoliticos enfrentados que resuelven a su manera el eje de relaciones entre individuos, gobiernos y compañias, en dos escenarios diferentes. Por un lado, Occidente, donde pueden encontrarse a grandes compañias como Google y Facebook con importantes acusaciones de competencia desleal y manipulación; y, por otro lado, China, donde las autoridades "protegen" a sus 800 millones de internautas en lo que puede entenderse como un peligroso modelo de integración vertical.

Finalmente, en nuestra Sección Acta Tántrica, presentamos dos investigaciones en las que se explora como la filosofía tántrica puede a la vez ser una herramienta de liberación intelectual y cómo conlleva resultados poco conocidos por la medicina clásica en relación a la salud sexual. En el artículo, (Bauer, 2019 c) "Espacio transicional: Una apertura del reino experimental más allá de la mente" el autor describe como de la fusión de las doctrinas del budismo temprano -influenciado por Friedrich Majer- con la filosofia occidental, Schopenhauer elabora su propio sistema de exploración fenomenológica y su concepto de impulso "Trieb» sin objeto -que es la base de la doctrina psicoanalitica de pulsión en Freud-. Aborda las coincidencias ontológicas de nuestra comprensión fenomenológica existencial contemporánea y la praxis tántrica Dzogchen de los siglos VIII y IX. El artículo muestra cómo esta comprensión tántrica ilumina el pensamiento existencialista que atraviesa la 
fenomenología trascendental de Husserl y más tarde la fenomenología ontológica de Heidegger y Merleau-Ponty. El artículo también revela cómo estas dos modalidades de psicoterapia fenomenológica existencial y praxis tántrica convergen en el proceso experiencial de auto liberación. El trabajo también describe cómo una experiencia que es de fácil acceso se convirtió en el mundo espiritual patriarcal en algo propio de unos pocos «iluminados" como producto corporativo o marca ofreciendo, así, con este artículo, el más oculto secreto del tantra-Mediante la metáfora de kundalini explica como una persona puede discriminar a quién desea sexualizar. Esta discriminación ayuda a las personas a ser menos atadas y dominadas por su incansable impulso hacia la descarga sexual implacable y compulsiva-.

Por último, en esta sección, presentamos "Tantra y los límites del potencial femenino en la respuesta sexual" (Sayin, 2019) Este artículo explica algunos de los nuevos hallazgos sobre la sexualidad femenina, la Respuesta Sexual Expandida (RSE) y los orgasmos prolongados-ampliados en comparación con las descripciones de las antiguas filosofias tántricas y taoístas. En esta revisión se presentan descripciones detalladas de estos fenómenos en el marco de investigaciones clínicas actuales.

Oscar R. Gómez

Director 


\section{Referencias}

Basu, R. (2019). Soluciones al sufrimiento de los oprimidos: De la Revolución Cultural a la Evolución Cultural. Revista Científica Arbitrada de la Fundación MenteClara, 4(2), 133-152. DOI: https://doi.org/10.32351/rca.v4.2.101

Bauer, R. (2019). La mente como conocedora de las formas y la conciencia como conocedora del Ser: Una visión fenomenológica. Revista Científica Arbitrada de la Fundación MenteClara, 4(2), 47-53. DOI: https://doi.org/10.32351/rca.v4.2.86

Bauer, R. (2019). Ontología de la dicha: Una fenomenología. Revista Científica Arbitrada de la Fundación MenteClara, 4(2), 55-70. DOI: https://doi.org/10.32351/rca.v4.2.87

Bauer, R. (2019). Espacio transicional: Una apertura del reino experimental más allá de la mente. Revista Científica Arbitrada de la Fundación MenteClara, 4(2), 167-201. DOI: https://doi.org/10.32351/rca.v4.2.88

Bose, A. (2019). Hacia una poética comparativa de Buda, Kabir y Gurú Nanak desde una perspectiva democrática secular. Revista Científica Arbitrada de la Fundación MenteClara, 4(2), 19-44. DOI: https://doi.org/10.32351/rca.v4.2.85

Dapena Botero, J. (2019). En busca de un posible tratamiento psicoanalítico de la ludopatía. Revista Científica Arbitrada de la Fundación MenteClara, 4(2), 111-131. DOI: https://doi.org/10.32351/rca.v4.2.95

Duman, E. (2019). Implementación del concepto de angst de Heidegger en el problema budista sobre el sufrimiento: Cómo el discurso budista genera dificultades epistemológicas y cómo abordarlo con la ontología occidental. Revista Científica Arbitrada de la Fundación MenteClara, 4(2), 7-17. DOI: https://doi.org/10.32351/rca.v4.2.84

López Alurralde, J., \& Becerra, G. (2019). Modelos sociopolíticos en disputa. El caso del bloqueo a Huawei. Revista Científica Arbitrada de la Fundación MenteClara, 4(2), 153-163. DOI: https://doi.org/10.32351/rca.v4.2.105

Moss-Racusin, C. A., Dovidio, J. F., Brescoll, V. L., Graham, M. J., \& Handelsman, J. (2012). Science faculty's subtle gender biases favor male students. Proceedings of the National Academy of Sciences, 109(41), 1647416479. DOI: https://doi.org/10.1073/pnas.1211286109

Sayin, Ü. (2019). Tantra y los límites del potencial femenino en la respuesta sexual. Revista Científica Arbitrada de la Fundación MenteClara, 4(2), 203-243. DOI: https://doi.org/10.32351/rca.v4.2.104

Simkin, H., Rubio, P., di Puglia, G., \& Preuss, M. (2019). Religiosidad y espiritualidad en el marco del modelo de los cinco factores de la personalidad. Revista Científica Arbitrada de la Fundación MenteClara, 4(2), 73109. DOI: https://doi.org/10.32351/rca.v4.2.89 